\title{
Intensive Care Therapy for Patients with Advanced Liver Diseases
}

\author{
Antonios Katsounas Ali Canbay \\ Department for Gastroenterology, Hepatology and Infectious Diseases, University Hospital Magdeburg, Magdeburg, Germany
}

\section{Keywords}

Acute-on-chronic liver failure, ACLF - Cirrhosis .

Critical care $\cdot$ Immune dysfunction - Organ dysfunction

\section{Summary}

Decompensated cirrhosis is characterized by high hospitalization rates and costs, frequent readmissions, and poor short-term survival. Patients admitted to the hospital with acute variceal bleeding and/or hepatic encephalopathy and/or renal dysfunction are at serious risk for developing infection and/or sepsis; in turn, this renders them highly susceptible to the development of multi-system organ failure. The lack of standardized intensive care unit management protocols in patients with cirrhosis along with only few data reports from longitudinal clinical trials makes it difficult for hepatologists and critical care specialists to provide uniform evidence for clinical practice that could safely consolidate favorable outcomes such as lower hospitalization rates and/or mortality. Based on a rigorous online search of the scientific literature as well as a longtime clinical experience of the authors in the field of hepatology and critical care medicine, this work represents a focused effort to elucidate the specific bio-morbidity of advanced liver diseases in relation to the aforementioned challenges in clinical management. Further meta-analyses and/or systematic reviews are needed to enable clinicians to develop more effective strategies to bridge patients with decompensated liver disease to recompensation or liver transplantation.

(C) 2018 S. Karger GmbH, Freiburg

\section{Introduction}

Patients with advanced liver diseases experience numerous complications such as:

- variceal bleeding (VB);

- hemostasis dysfunction;

- renal dysfunction;

- cardiovascular dysfunction;

- adrenal dysfunction;

- hepatic encephalopathy (HE);

- immune dysfunction;

- infection and/or sepsis.

These severe conditions lead to increasing hospitalization and/ or mortality rates of up to $50 \%$ within 5 years [1]. Approximately $10 \%$ of patients with liver cirrhosis, which are hospitalized due to acute decompensation, require intensive care therapy as they are (at serious risk for) developing (multi-)organ failure. Patients diagnosed with acute-on-chronic liver failure (ACLF) - as determined by the Chronic Liver Failure (CLIF) score along with its prognosis using the CLIF-ACLF score (www.clifconsortium.com) - show a significantly higher 28-day mortality rate compared to patients without ACLF $[2,3]$. ACLF is usually part of a multi-system organ failure (liver, kidney, central nervous system, coagulation, circulation, respiration) in patients with decompensated cirrhosis. Despite that, clinical management still lacks standardized treatment strategies. To date, intensive care therapy carried out on the basis of interaction between different disciplines, e.g. intensivists, hepatologists, nephrologists, transplant surgeons, and infectious disease specialists, is considered more promising with regard to more favorable clinical outcomes [4].

\section{Variceal Bleeding}

Approximately $60 \%$ of patients with decompensated liver cirrhosis have esophageal varices; in contrast, only $30 \%$ of patients

\section{KARGER}

() 2018 S. Karger GmbH, Freiburg

Fax +497614520714
PD Dr. Antonios Katsounas

Department for Gastroenterology, Hepatology and Infectious Diseases University Hospital Magdeburg

Leipziger Straße 44, 39120 Magdeburg, Germany

antonios.katsounas@med.ovgu.de 
with compensated liver disease face this complication. The incidence of VB amounts to approximately 5\% (unless varices are of medium or large size; in that case, VB is three times more frequent), with a recurrence rate of $60 \%$ within 2 years [5]. If measured by hard outcomes such as survival, clinicians should then be aware of a high VB-related mortality reaching from $20 \%$ during the acute episode up to over 50\% after 12 months [6]. Thus, it is crucial to strictly comply with evidence-based algorithms for the treatment of VB due to its significant impact on short- as well as longterm survival. In this light, VB should be preferably managed in the intensive care unit (ICU) (or at least the intermediate care unit) due to the imminent risk of re-bleeding and/or sepsis. As boosted fluid substitution may aggravate portal hypertension and provoke uncontrolled re-/bleeding, a restrictive transfusion strategy aimed at maintaining hemoglobin levels between 7 and $9 \mathrm{~g} / \mathrm{dl}$ (using a transfusion threshold for hemoglobin levels $<7 \mathrm{~g} / \mathrm{dl}$ ) should be applied. This strategy has been proven to be significantly associated with less re-bleeding episodes and a higher 6-week survival in comparison to liberal transfusion practices [7]. If unavoidable, endotracheal intubation for airway protection (against aspiration) should be performed prior to endoscopy; prevention of HE with non-absorbable disaccharides is considered controversial in VB; however, a recent randomized controlled trial (RCT) revealed that lactulose had a stronger clinical benefit compared to a placebo in these cases [8]. In order to prevent post-VB infection and/or sepsis, prophylactic administration of antibiotics has been proven essential [5]. In this regard, a Cochrane meta-analysis that evaluated twelve RCTs including patients with VB $(\mathrm{N}>1,200)$ has confirmed a reduction of bacterial infection and mortality rates (related to bacterial infection) as well as of re-bleeding rates and overall mortality in patients that received antibiotic prophylaxis [9]. In patients with advanced cirrhosis, i.e. Child-Pugh stage B and C, oral quinolones, e.g. norfloxacin, were shown to be effective but of inferior efficacy in comparison to intravenous ceftriaxone [10]. However, the use of prophylactic antibiotics should be adapted to the local epidemiological patterns of antibiotic resistance and to the individual patient's history of bacterial colonization/infection. Standard therapy for VB should combine endoscopic and pharmacological measures, as this combination has been proven to be superior to either single pharmacological or single endoscopic therapy [11]. Vasoactive drugs, such as terlipressin or somatostatin, should be administered as early as possible $[11,12]$. Endoscopic variceal ligation (EVL) should also be performed as soon as possible (i.e. within $12 \mathrm{~h}$ ) after VB. EVL is considered more effective when compared to sclerotherapy. Finally yet importantly, the use of transjugular intrahepatic portosystemic shunts (TIPS) is considered a rescue treatment for patients not responding to standard endoscopic and pharmacological therapy. Recently, early TIPS (to be performed within the first $72 \mathrm{~h}$ ) has been recommended in association with standard endoscopic and pharmacological therapy for cirrhotic patients with Child-Pugh stage B and active VB or Child-Pugh stage C with $<14$ points $[13,14]$. In case of severe bleeding with emerging shock, intensive care therapy should be aimed at i) stabilizing hemodynamics by holding systolic blood pressure at $80-100 \mathrm{~mm} \mathrm{Hg}$ and/or mean arterial pressure (MAP) $\geq 65 \mathrm{~mm} \mathrm{Hg}$ along with a heart rate $\leq 120 \mathrm{bpm}$; ii) restoring lactate clearance; iii) normalizing base excess and keeping ionized calcium levels $>1.0 \mathrm{mmol} / \mathrm{l}$; and iv) maintaining adequate urine output [15]. TIPS might also be considered in some cases with compensated cirrhosis. Indeed, due to portal hypertension, the mucosa of the colon is swollen; therefore, not only mucosal resorption can be impaired but also the gut microbiome can get out of balance, which then likely favors development of sarcopenia. Administration of blood coagulation components should be performed according to clinically evaluated replacement algorithms based on thromboelastography; this tool allows rapid differential diagnosis, assessment of platelet activity, and accurate monitoring of the effectiveness of therapy [16].

\section{Hemostasis Dysfunction}

In patients with advanced liver cirrhosis, low platelet counts along with attenuation/loss of their function, decreased concentrations of plasmatic procoagulant factors and/or vitamin $\mathrm{K}$, hypofibrinogenemia or reduced levels of protein $\mathrm{C}$ and $\mathrm{S}$ create an instable equilibrium between prothrombotic and profibrinolytic pathophysiology. In the plasma of cirrhotic subjects, increased levels of the tissue plasminogen activator (tPA) have been reported in contrast to reduced levels of factor XIII, plasminogen, and alpha2-antiplasmin $[17,18]$. These changes result from the fact that all proteins facilitating fibrinolysis are produced in the liver except for tPA and the plasminogen activator inhibitor 1. Viscoelastic tests, such as thromboelastography, provide evidence that patients with acute liver failure (ALF) or non-alcoholic steatohepatitis or cholestatic liver diseases are at risk for thrombotic complications based on a more prevalent hypercoagulability $[19,20]$. Although the role of viscoelastic testing for the management of these patients has not been sufficiently studied yet, international guidelines already recommend that administration of antifibrinolytics and/or replacement of procoagulant components should be performed with caution [4]. In absence of previous history and/or other risk factors for hemorrhagic complications [21, 22], low-molecular-weight heparin likely represents the safest choice of systemic anticoagulation in cirrhotic patients with extensive portal vein (and/or mesenteric) thrombosis without increasing the risk for VB [23]. However, clinicians should always be aware of the fact that (despite decreased antithrombin III concentrations) its anticoagulation effect in vivo remains practically unaffected [24]. More aspects of 'hemostasis dysregulation' are shown by Drolz et al. [25] in this focus issue of VISCERAl Medicine.

\section{Renal Dysfunction}

Patients with advanced liver disease, especially those with severe alcoholic steatohepatitis, are prone to develop acute renal failure, and, thus, inevitably progress to ACLF along with multi-system organ failure [26, 27]. Renal failure occurs in approximately $40-$ 
Table 1. Criteria required for diagnosis of hepatorenal syndrome (HRS)

\begin{tabular}{ll}
\hline 1 & Confirmed cirrhosis along with ascites \\
\hline 2 & Exclusion of circulatory shock \\
\hline 3 & No current or recently interrupted treatment with nephrotoxic substances \\
\hline 4 & Serum creatinine $>1.5 \mathrm{mg} / \mathrm{dl}(133 \mathrm{mmol} / \mathrm{l})$ \\
\hline 5 & $\begin{array}{l}\text { No improvement of serum creatinine (= decrease to } \leq \text { upper normal } \\
\text { limit }) \geq 48 \text { h post-withdrawal of diuretic drugs and despite sufficient } \\
\text { volume expansion with albumin ( } 1 \text { g/kg of body weight/day up to } \\
\text { maximally } 100 \text { g/day) }\end{array}$ \\
\hline $6 \quad \begin{array}{l}\text { Exclusion of parenchymal kidney disease as diagnosed via } \\
\text { microhematuria ( }>50 \text { red blood cells per high power field), proteinuria } \\
(>500 \text { mg/24 h) and/or abnormal renal ultrasound examination }\end{array}$ \\
\hline
\end{tabular}

$50 \%$ of cirrhotic patients admitted to the ICU, most commonly triggered by hypovolemia, systemic bacterial infections, acute tubular necrosis, or hepatorenal syndrome (HRS) [26]. Type-1 HRS is hallmarked by increasing serum creatinine levels of $>2.5 \mathrm{mg} / \mathrm{dl}$ within 2 weeks [28]. Type-2 HRS predominantly emerges in nonazotemic cirrhotic patients with refractory ascites and moderate but relatively steady renal insufficiency. Beyond a spontaneous bacterial peritonitis (SBP) and/or other bacterial infections, an acute hepatitis flare-up, gastrointestinal bleeding episodes, and/or major surgical interventions usually render patients with cirrhosis more susceptible to the development of type-1 HRS (table 1).

Type-1 HRS, which is associated with severe systemic inflammation (SI), has a poor prognosis with a median survival time of 15 days. Arterial vasodilation caused by SI leads to progressive attenuation of left ventricular function, which in turn aggravates circulatory dysfunction in patients with liver cirrhosis. Vasoconstriction of the kidneys and other extra-splanchnic vascular compartments is the result of a compensatory mechanism to maintain normal (arterial) blood pressure. Ongoing renal vasoconstriction downregulates renal perfusion and diminishes glomerular filtration rate [29, 30]. In contrast, portal hypertension is associated with local release of nitric oxide (NO) and other vasodilatory molecules in the arterial splanchnic circulation of cirrhotic patients. These changes favor the development of a hyperdynamic circulation (i.e. increased cardiac output along with tachycardia) as a physiological compensatory reaction to arterial hypotension. In end-stage liver disease, severe arterial hypotension along with terminal renal vasoconstriction and complete deterioration in effective arterial blood flow lead to HRS. Surprisingly, HRS does not strongly affect the 3 -year survival rate of patients with HRS (60-65\%) undergoing liver transplantation (LTx) compared to those without HRS (70$80 \%)$. Although LTx remains the most effective treatment for patients with HRS, most patients with type-1 HRS cannot be successfully bridged to transplantation because of a short median survival time. In such cases, vasoconstrictory drugs (especially terlipressin and noradrenaline) applied in combination with intravenous albumin may be able to cure HRS with a possibility of $40-50 \%[31,32]$. Meanwhile, published reports on the use of other vasoconstrictors such as midodrine are equally promising; however, terlipressin, as a well-studied drug, still represents a first-line therapy in clinical routine and should be administered in increasing dosage beginning with $0.5 \mathrm{mg} / 4 \mathrm{~h}$. In the case that serum creatinine does not improve by $>30 \%$ within $72 \mathrm{~h}$, the infusion rate should be increased by $100 \%$. A meta-analysis evaluating the efficacy and safety of noradrenaline versus terlipressin confirmed comparable results for both vasoconstrictors; however, noradrenaline had the undisputable advantage of much lower daily costs in comparison to terlipressin [33]. As stated in table 1, albumin should be co-administered starting with an initial dose of $1 \mathrm{~g} / \mathrm{kg}$ of body weight up to a maximum of $100 \mathrm{~g} /$ day. To date, the use of TIPS still lacks evidence for (at least) an equally good outcome when compared to vasoconstrictors. Furthermore, there is significant proof suggesting that cardiac dysfunction aggravates HRS [34, 35].

\section{Cardiovascular Dysfunction}

As already mentioned, progressive loss of the left ventricular function and reduction of cardiac output, which develops in decompensated cirrhosis, contributes to circulatory dysfunction. Recent data provided significant evidence for the role of tumor necrosis factor alpha(TNF- $\alpha$ )-related activation of the inducible NO synthase in cardiac tissue, suggesting that inflammation is causally involved in diminishing cardiac ejection fraction in cirrhosis [36]. In this light, it seems plausible why activation of the sympathetic nervous system, which attenuates intestinal immunity by altering the gut microbiota, contributes to progression of cardiovascular dysfunction [36]. Bacterial translocation silently activates the intestinal immune system, resulting in production and release of vasodilatory molecules such as NO. During progression of the disease, the lack of arterial effective circulating volume along with the consecutive activation of the renin-angiotensin system and the continuous non-osmotic release of the antidiuretic hormone from the pituitary cause an excessive retention of free water that leads to dilutional hyponatremia and advanced ascites formation [37]. Interestingly, albumin infusion is associated with a more favorable outcome in comparison to hydroxyethyl starch with regard to improving hemodynamic conditions including peripheral vascular resistance, left ventricular function, and cardiac output in patients with non-severe SBP [38]. However, despite conflicting data [39], a more recent study failed to prove that albumin co-administered with crystalloids could improve overall survival although its use was associated with higher MAP and lower heart rate values when compared to single resuscitation with crystalloid in cirrhotic patients with SBP [40]. Of note, albumin has been proven capable of restoring endothelial function due to its attributes as a detoxifying and/or antioxidant substance. In addition, noradrenaline is strongly recommended as the vasopressor of choice while vasopressin should no longer be considered as a proper initial therapy in cirrhotic patients with circulatory failure. In case of a resuscitation-resistant hypoperfusion, dobutamine could be added to norepinephrine, especially if myocardial dysfunction is suspected. 


\section{Adrenal Dysfunction}

Patients with liver cirrhosis are at risk of developing adrenal insufficiency (AI); this complication is favored by reduced cholesterol synthesis and persistent endotoxemia along with coagulopathy that can cause severe damage in adrenal parenchyma [41, 42]. It is well known that cortisol is capable of regulating i) vascular tonus and permeability; ii) endothelial integrity; iii) distribution of water in body compartments; iv) suppression of cytokines such as TNF- $\alpha$ as well as interleukin(IL)-1 and IL-6 [42]. Thus, AI is considered as causally associated with life-threatening conditions such as multi-system organ failure due to refractory (septic) shock and bears a high risk of hospital mortality [41, 42]. Patients with advanced cirrhosis experiencing major stress, e.g. sepsis/septic shock, VB, or extensive surgery (including LTx), likely develop an AI defined as the difference $\Delta<9 \mu \mathrm{g} / \mathrm{l}$ or $250 \mathrm{nmol} / \mathrm{l}$ between peak and basal cortisol level after standard challenge with adrenocorticotropic hormone or random serum total cortisol $<10 \mu \mathrm{g} / \mathrm{dl}$ or 276 $\mathrm{nmol} / \mathrm{l}[41,42]$. Previous studies that predominantly included noncirrhotic patients with septic shock revealed distinct data regarding the impact of corticosteroids on mortality [43]. Two more recent trials enrolling critically ill patients with cirrhosis and severe sepsis/septic shock concluded that two-thirds of all cases developed an $\mathrm{AI}$ and that these patients showed a higher hospital mortality rate compared to patients without AI [44, 45]. Moreover, four independent parameters that predicted AI were identified: i) MAP, ii) vasopressor dependency, iii) bacteremia, and iv) serum bilirubin [46]. Moreover, patients with cirrhosis and septic shock who developed an AI exhibited a more severe hepatorenal and circulatory impairment and had a higher frequency of bacteremia and leukocyte counts. Retrospectively, administration of hydrocortisone was associated with improved in-hospital survival [44]. In a further effort to prospectively evaluate this finding, another trial could also confirm hemodynamic improvement; however, this study found that treated patients also had a higher risk for shock relapse (after interruption of therapy with corticosteroids) and gastrointestinal bleeding as well as severe hyperglycemia [46]. Hyperglycemia reportedly impairs neutrophil function and favors apoptosis and prothrombotic activity. Unfortunately, to date, there are no trials designed/conducted to define the optimal target range of glucose levels in the blood of critically ill patients with cirrhosis. Current literature recommends a glucose target range of $140-180 \mathrm{mg} / \mathrm{dl}$ for these patients [47]. This statement is based on results of an RCT that enrolled critically ill patients ( $\mathrm{N}>6,000$; of those, $30 \%$ had an underlying liver disease but no confirmed cirrhosis). This RCT detected a higher mortality rate in patients treated intensively (with a glucose target range between 81 and $108 \mathrm{mg} / \mathrm{dl}$ ) compared to those with a glucose target range between 140 and $180 \mathrm{mg} / \mathrm{dl}$ [48].

\section{Hepatic Encephalopathy}

$\mathrm{HE}$ is a neurological syndrome often related to portosystemic shunts that can affect patients with ALF and can deteriorate quality
Table 2. Other causes to be excluded versus hepatic encephalopathy (HE)

Other causes to be excluded versus $H E$

Respiratory deterioration with oxygenation disorder and/or decarboxylation disorder

Acidosis

Hypoglycemia

Uremia

Electrolyte disorders

Myxedema coma

Septic encephalopathy

Stroke-related pre-/seizure, postictal confusion

Intracerebral hemorrhage

Wernicke-Korsakoff syndrome

Alcohol withdrawal delirium (delirium tremens)

Central nervous system drug intoxication

Triggers for $\mathrm{HE}$

Sepsis

Dehydration

Gastrointestinal bleeding

Consumption of animal-derived protein

Constipation, bowel obstruction, or ileus

Poor compliance to therapy with lactulose and/or antibiotics

Transjugular intrahepatic portosystemic shunt

Spontaneous bacterial peritonitis or other (site of) infection

Aggravated portal hypertension

Superimposed liver injury

Primary liver carcinoma and/or intrahepatic metastasis

Backward heart failure

of life and/or increase mortality in subjects with cirrhosis [49]. It is also characterized by a progressive but potentially reversible cerebral dysfunction with a wide symptomatology ranging from neuropsychiatric to motor symptoms and from slightly altered cognitive function to coma. Apparently, nystagmus, seizures, and transient focal deficits are less common [50]. Once the first episode of acute HE occurs, the probability of survival without LTx drops to $42 \%$ at 1 year and $23 \%$ at 3 years [51]. The in-hospital mortality rate for patients suffering from severe HE in the ICU is 35\%, and the 1 -year mortality rate is approximately $55 \%$ [52]. Although not all mechanisms driving the pathogenesis of $\mathrm{HE}$ are clear yet, the leading hypothesis remains the lack or failure of functional hepatic pathways to eliminate nitrogen-derived products (e.g. ammonia) [53]. Apart from the presence of a liver disease, diagnosis of HE also requires the exclusion of other causes of brain dysfunction, especially in patients that do not promptly respond to anti-HE therapy. Despite controversial reports, ammonia (measured in the blood) may be a reliable marker for HE in patients with advanced liver diseases, as long as clinicians remain aware of the significant interindividual variation in its blood concentrations; also, $10 \%$ of patients with overt HE exhibit blood ammonia values within the normal range. Meanwhile, magnetic resonance imaging is capable of detecting alterations typical for HE, such as a decrease of brain volume and/or paramagnetic spot signals in the basal ganglia area. Diagnostics based on computed tomography may also be useful to 
Table 3. Anti-hepatic encephalopathy medications

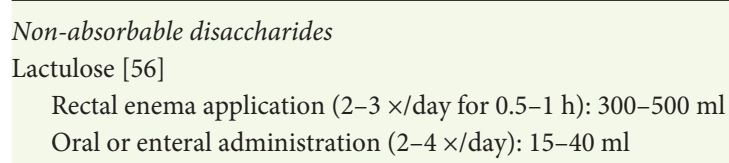

Antibiotics

Rifaximin [57]

Oral or enteral administration $(2 \times /$ day $): 550 \mathrm{mg}$

Branched-chain amino acids (BCAA)

L-ornitine L-aspartate [58]

IV supplementation for 3-7 days: 20-40 g/24 h

Oral supplementation: 3-6 g/day

Currently under investigation

Flumazenil (synthetic benzodiazepine antagonist) [59]

Pre-/probiotics [60]

Fecal microbiome transfer [61]

confirm or rule out infarction, hemorrhage, abscess formations, or tumors. Furthermore, an electroencephalogram may detect some quite common but usually not specific wave patterns for HE [50]. According to the West Haven classification, severity of HE can be assessed by using four grades. However, based on the (more recent) SONIC classification, which has been introduced by the International Society for Hepatic Encephalopathy and Nitrogen Metabolism, minimal and grade $1 \mathrm{HE}$ represent a 'covert HE' while the 'clinically apparent HE' encompasses grade 2-4 HE [54]. The clinical management of patients with $\mathrm{HE}$ requires a wide and rigorous differential screening for other causes of encephalopathy and identification of triggers for $\mathrm{HE}$ (table 2). Specific therapies for HE (table 3) are often initiated along with treatment of the triggering factors such as gastrointestinal bleeding, toxic medications, electrolyte disorders, and renal dysfunction (table 2) [55].

Cirrhotic patients with HE should have a daily protein intake of $1.2-1.5 \mathrm{~g} / \mathrm{kg}$ body weight in order to prevent further sarcopenia and thus progress to cachexia. Patients with severe HE should be supplemented with branched-chain amino acids and only low amounts of aromatic amino acids to improve/prevent HE symptoms [62].

\section{Immune Dysfunction}

Both bacterial antigens, e.g. pathogen-associated molecular patterns (PAMP), and endogenous molecules expressed upon cell injury, e.g. damage-associated molecular patterns (DAMP), can trigger SI. Interaction between PAMP or DAMP and the innate immune system via specific receptors drives the systemic release of inflammatory mediators. An excessive inflammatory activity also seems to play a crucial role in acute alcoholic hepatitis and/or other settings of acute liver damage [36]. In addition, a 'leaky gut' favoring translocation of bacteria towards the bloodstream along with a gradually impaired hepatic clearance capacity for bacterial anti- gens, such as lipopolysaccharide (LPS) or endotoxin, may induce activation of toll-like receptor (TLR) pathways, and, thus, further enhance SI that accelerates fibrogenesis and progression to liver cirrhosis [63]. This, in turn, boosts cytokine secretion along with an ongoing synthesis of reactive oxygen species (ROS), thereby forming a vicious circle in the development of intestinal inflammation and tissue hyperpermeability $[64,65]$. In this light, it is reasonable to assume that some beneficial effects of albumin administration in cirrhotic patients with SBP and/or type-1 HRS might be largely attributable to its anti-inflammatory and antioxidative stress properties [36]. This hypothesis gains considerable support by recent studies in patients with cirrhosis and SBP reporting that combined treatment with intravenous albumin and an antibiotic reduces the risk for renal dysfunction/failure and mortality in comparison to therapy with a single antibiotic [39]. Last but not least, clinical investigations, which clearly identified white blood cell count and C-reactive protein (CRP) as independent predictors of in-hospital survival, add great evidence to the prognosis-relevant role of SI $[64,65]$. More information on 'immune dysregulation' is given by Lange and Moreau [66] in this focus issue of VISCERAL Medicine.

\section{Infection and/or Sepsis}

Liver cirrhosis is a serious risk factor for hospitalization due to infection and for sepsis-related mortality; in patients with advanced liver disease, prevalence of in-hospital infections amounts to $25-35 \%$ and to approximately $60 \%$ in patients admitted to the ICU [67-69]. In comparison to other ICU patient groups, the presence of liver cirrhosis renders subjects acquiring infections more susceptible to multi-system organ failure due to septic shock [69]. Overall (listing the types of infections from most to least frequent), cirrhotic patients are prone to develop SBP, urinary tract infections, pneumonia, bacteremia, and skin/soft tissue infections [70]. In the ICU, pneumonia represents the most common septic trigger observed in association with liver cirrhosis; here, fungal infections represent a particularly life-threatening scenario [67, 68, 71]. Infection and pathogen surveillance programs identified gram-positive cocci as the most likely cause of nosocomial infections in association with invasive diagnostic and/or therapeutic procedures [6769]. In contrast, gram-negative bacilli are responsible for approximately $60 \%$ of all community-acquired infections $[67,68,71]$. During the past 10 years, prevalence of nosocomial infections involving multiresistant bacteria has increased from $10 \%$ to $20-25 \%$. In the same period, multiresistant strains could be detected in up to $40 \%$ of cirrhotic patients diagnosed with nosocomial infections; this trend likely provides a rational explanation for the sharp rise in mortality, i.e. a two-fold increase, within the last decade $[67,68]$.

Based on Sepsis-3 criteria, sepsis is defined as severe organ dysfunction triggered by a dysregulated biological response to infection [72-74]. Organ dysfunction is defined as an increase of $\geq 2$ points in the Sequential Organ Failure Assessment (SOFA) score [72-74] (table 4). 
Table 4. SOFA score according to Vincent et al. [76]

\begin{tabular}{|c|c|c|c|c|c|}
\hline & \multicolumn{5}{|l|}{ SOFA score } \\
\hline & 0 & 1 & 2 & 3 & 4 \\
\hline \multicolumn{6}{|l|}{ Respiration } \\
\hline $\mathrm{PaO}_{2} / \mathrm{FIO}_{2}, \mathrm{~mm} \mathrm{Hg}(\mathrm{kPa})$ & $\leq 400(53.3)$ & $<400(53.3)$ & $<300(40)$ & $\begin{array}{l}<200(26.7) \text { with } \\
\text { respiratory support }\end{array}$ & $\begin{array}{l}<100(13.3) \text { with } \\
\text { respiratory support }\end{array}$ \\
\hline \multicolumn{6}{|l|}{ Coagulation } \\
\hline Platelets, $\times 10^{3} / \mu \mathrm{l}$ & $\geq 150$ & $<150$ & $<100$ & $<50$ & $<20$ \\
\hline \multicolumn{6}{|l|}{ Liver } \\
\hline Bilirubin, mg/dl ( $\mu \mathrm{mol} / \mathrm{l})$ & $<1.2$ & $1.2-1.9(20-32)$ & $2.0-5.9(33-101)$ & $6.0-11.9(102-204)$ & $>12.0(204)$ \\
\hline Cardiovascular & $\begin{array}{l}\mathrm{MAP} \geq \\
70 \mathrm{~mm} \mathrm{Hg}\end{array}$ & $\begin{array}{l}\text { MAP < } \\
70 \mathrm{~mm} \mathrm{Hg}\end{array}$ & $\begin{array}{l}\text { dopamine }<5 \text { or } \\
\text { dobutamine (any dose) }\end{array}$ & $\begin{array}{l}\text { dopamine } 5.1-15 \text { or } \\
\text { epinephrine } \leq 0.1 \text { or } \\
\text { norepinephrine } \leq 0.1\end{array}$ & $\begin{array}{l}\text { dopamine }>15 \text { or epinephrine } \\
>0.1 \text { or norepinephrine }>0.1\end{array}$ \\
\hline \multicolumn{6}{|l|}{ Central nervous system } \\
\hline Glasgow Coma Scale score ${ }^{\mathrm{a}}$ & 15 & $13-14$ & $10-12$ & $6-9$ & $<6$ \\
\hline \multicolumn{6}{|l|}{ Renal } \\
\hline Creatinine, mg/dl $(\mu \mathrm{mol} / \mathrm{l})$ & $<1.2(110)$ & $\begin{array}{l}1.2-1.9 \\
(110-170)\end{array}$ & $\begin{array}{l}2.0-3.4 \\
(171-299)\end{array}$ & $\begin{array}{l}3.5-4.9 \\
(300-440)\end{array}$ & $>5.0(\geq 441)$ \\
\hline Urine output, $\mathrm{ml} / \mathrm{d}$ & & & & $<500$ & $<200$ \\
\hline \multicolumn{6}{|c|}{$\begin{array}{l}{ }^{\mathrm{a}} \text { Glasgow Coma Scale scores range from } 3 \text { to } 15 \text {; higher score values indicate better neurological function. } \\
\mathrm{FIO}_{2}=\text { Fraction of inspired oxygen; } \mathrm{MAP}=\text { mean arterial pressure; } \mathrm{PaO}_{2}=\text { partial pressure of oxygen. } \\
\text { Catecholamine doses are given as } \mu \mathrm{g} / \mathrm{kg} / \mathrm{min} \text { for at least } 1 \mathrm{~h} \text {. }\end{array}$} \\
\hline
\end{tabular}

In patients with liver cirrhosis and sepsis, intensive care therapy is aimed to restore organ perfusion (as described above) along with fast identification and elimination of the infectious site(s) and trigger(s). More information on this particular aspect can be found in the contribution by Engelmann and Berg [75] in this focus issue of Visceral Medicine.

\section{Conclusion}

Due to an increased susceptibility and vulnerability to multisystem organ failure, ICU patients with liver diseases require special attention and their management demands effective collabora- tion between intensive care physicians, hepatologists, kidney and infectious disease specialists, and transplant experts. In the last decade, our knowledge in the overlapping area of hepatology and critical care expanded and strategies for diagnostics and therapeutic interventions improved. In the future, more controlled trials will be able to drive the evolution of our skills in this field from cutting-edge medicine to standardized clinical practice.

\section{Disclosure Statement}

The authors have no conflicts of interest to disclose regarding this review.

\section{References}

1 Pere G, et al: Compensated cirrhosis: natural history and prognostic factors. Hepatology 1987;7:122-128.

2 Jalan R, et al: Bacterial infections in cirrhosis: a position statement based on the EASL Special Conference 2013. J Hepatol 2014;60:1310-1324.

3 Jalan R, et al: Acute-on chronic liver failure. J Hepatol 2012;57:1336-1348.

4 Nadim MK, et al: Management of the critically ill patient with cirrhosis: a multidisciplinary perspective. J Hepatol 2016;64:717-735.

5 Bosch J, et al: The management of portal hypertension: rational basis, available treatments and future options. J Hepatol 2008;48(suppl 1):S68-92.
6 Garcia-Tsao G, Bosch J, Groszmann RJ: Portal hypertension and variceal bleeding - unresolved issues. Summary of an American Association for the Study of Liver Diseases and European Association for the Study of the Liver single-topic conference. Hepatology 2008; 47:1764-1772.

7 Villanueva $\mathrm{C}$, et al: Transfusion strategies for acute upper gastrointestinal bleeding. N Engl J Med 2013; 368:11-21.

8 Sharma P, et al: Prophylaxis of hepatic encephalopathy in acute variceal bleed: a randomized controlled trial of lactulose versus no lactulose. J Gastroenterol Hepatol 2011;26:996-1003.
9 Chavez-Tapia NC, et al: Meta-analysis: antibiotic prophylaxis for cirrhotic patients with upper gastrointestinal bleeding - an updated Cochrane review. Aliment Pharmacol Ther 2011;34:509-518.

10 Fernandez J, et al: Norfloxacin vs ceftriaxone in the prophylaxis of infections in patients with advanced cirrhosis and hemorrhage. Gastroenterology 2006;131: 1049-1056; quiz 1285.

11 Banares R, et al: Endoscopic treatment versus endoscopic plus pharmacologic treatment for acute variceal bleeding: a meta-analysis. Hepatology 2002;35:609615. 
12 Sarin SK, Sharma P: Terlipressin: an asset for hepatologists! Hepatology 2011;54:724-728.

13 Garcia-Pagan JC, et al: Early use of TIPS in patients with cirrhosis and variceal bleeding. $\mathrm{N}$ Engl J Med 2010;362:2370-2379.

14 Garcia-Pagán JC, et al: Use of early-TIPS for high-risk variceal bleeding: results of a post-RCT surveillance study. J Hepatol 2013;58:45-50.

15 Johansson PI, Ostrowski SR, Secher NH: Management of major blood loss: an update. Acta Anaesthesiol Scand 2010;54:1039-1049.

16 Bedreli S, et al: Management of acute-on-chronic liver failure: rotational thromboelastometry may reduce substitution of coagulation factors in liver cirrhosis. Gut 2016;65:357-358

17 Caldwell SH, et al: Coagulation disorders and hemostasis in liver disease: pathophysiology and critical assessment of current management. Hepatology 2006;44: 1039-1046.

18 Lisman T, Leebeek FWG, de Groot PG: Haemostatic abnormalities in patients with liver disease. J Hepatol 2002;37:280-287.

19 Krzanicki D, Sugavanam A, Mallett S: Intraoperative hypercoagulability during liver transplantation as demonstrated by thromboelastography. Liver Transpl 2013;19:852-861.

20 Ben-Ari Z, et al: Hypercoagulability in patients with primary biliary cirrhosis and primary sclerosing cholangitis evaluated by thrombelastography. J Hepatol 1997;26:554-559.

21 Francoz C, et al: Splanchnic vein thrombosis in candidates for liver transplantation: usefulness of screening and anticoagulation. Gut 2005;54:691-697.

22 Englesbe MJ, et al: Portal vein thrombosis and liver transplant survival benefit. Liver Transpl 2010;16:9991005.

23 Weeder PD, Porte RJ, Lisman T: Hemostasis in liver disease: implications of new concepts for perioperative management. Transfus Med Rev 2014;28:107-113.

24 Senzolo M, et al: Increased anticoagulant response to low-molecular-weight heparin in plasma from patients with advanced cirrhosis. J Thromb Haemost 2012;10: 1823-1829.

25 Drolz A, Ferlitsch A, Fuhrmann V: Management of coagulopathy during bleeding and invasive procedures in patients with liver failure. Visc Med 2018;34:10.1159/ 000491106.

26 Carvalho GC, et al: Causes of renal failure in patients with decompensated cirrhosis and its impact in hospital mortality. Ann Hepatol 2012;11:90-95.

27 Cholongitas E, et al: Cirrhotics admitted to intensive care unit: the impact of acute renal failure on mortality. Eur J Gastroenterol Hepatol 2009;21:744-750.

28 Sola-Vera J, et al: Randomized trial comparing albumin and saline in the prevention of paracentesis-induced circulatory dysfunction in cirrhotic patients with ascites. Hepatology 2003;37:1147-1153.

29 Ginès P, et al: Hepatorenal syndrome. Lancet 2003. 362:1819-1827.

-30 Salerno F, et al: Diagnosis, prevention and treatment of hepatorenal syndrome in cirrhosis. Gut 2007;56:13101318.

31 Martín-Llahí M, et al: Terlipressin and albumin vs albumin in patients with cirrhosis and hepatorenal syndrome: a randomized study. Gastroenterology 2008; 134:1352-1359.

32 Sanyal AJ, et al: A randomized, prospective, doubleblind, placebo-controlled trial of terlipressin for type 1 hepatorenal syndrome. Gastroenterology 2008;134: 1360-1368.
3 Singh V, et al: Noradrenaline vs. terlipressin in the treatment of hepatorenal syndrome: a randomized study. J Hepatol 2012;56:1293-1298.

$34 \mathrm{Krag}$ A, et al: Low cardiac output predicts development of hepatorenal syndrome and survival in patients with cirrhosis and ascites. Gut 2010;59:105-110.

35 Ruiz-del-Arbol L, et al: Circulatory function and hepatorenal syndrome in cirrhosis. Hepatology 2005; 42 : 439-447. 36 Arroyo V, García-Martinez R, Salvatella X: Human
serum albumin, systemic inflammation, and cirrhosis. J Hepatol 2014;61:396-407.

37 Pere G, et al: Hyponatremia in cirrhosis: from patho genesis to treatment. Hepatology 1998;28:851-864.

38 Fernández J, et al: Effect of intravenous albumin on systemic and hepatic hemodynamics and vasoactive neurohormonal systems in patients with cirrhosis and spontaneous bacterial peritonitis. J Hepatol 2004;41: 384-390.

39 Sort P, et al: Effect of intravenous albumin on renal impairment and mortality in patients with cirrhosis and spontaneous bacterial peritonitis. N Engl J Med 1999;341:403-409.

40 Caironi P, et al: Albumin replacement in patients with severe sepsis or septic shock. N Engl J Med 2014;370: 1412-1421.

41 Acevedo J, Fernández J: New determinants of prognosis in bacterial infections in cirrhosis. World J Gastroenterol 2014;20:7252-7259.

42 Trifan A, Chiriac S, Stanciu C: Update on adrenal insufficiency in patients with liver cirrhosis. World J Gastroenterology 2013;19:445-456.

43 Dellinger RP, et al: Surviving Sepsis Campaign: international guidelines for management of severe sepsis and septic shock, 2012. Crit Care Med 2013;41:580637.

44 Fernández J, et al: Adrenal insufficiency in patients with cirrhosis and septic shock: effect of treatment with hydrocortisone on survival. Hepatology 2006;44: 1288-1295.

45 Tsai $\mathrm{MH}$, et al: Adrenal insufficiency in patients with cirrhosis, severe sepsis and septic shock. Hepatology 2006;43:673-681.

46 Arabi YM, et al: Low-dose hydrocortisone in patients with cirrhosis and septic shock: a randomized controlled trial. CMAJ 2010;182:1971-1977.

47 Ginès P, et al: Management of critically-ill cirrhotic patients. J Hepatol 2012;56:S13-S24.

48 NICE-SUGAR Study Investigators; Finfer S, et al: Hypoglycemia and risk of death in critically ill patients. $\mathrm{N}$ Engl J Med 2012;367:1108-1118.

49 Vilstrup H, et al: Hepatic encephalopathy in chronic liver disease: 2014 Practice Guideline by the American Association for the Study of Liver Diseases and the European Association for the Study of the Liver. Hepatology 2014;60:715-735.

50 Córdoba J: New assessment of hepatic encephalopathy. J Hepatol 2011;54:1030-1040.

51 Stewart CA, et al: Hepatic encephalopathy as a predictor of survival in patients with end-stage liver disease. Liver Transpl 2007;13:1366-1371.

52 Fichet J, et al: Prognosis and 1-year mortality of intensive care unit patients with severe hepatic encephalop athy. J Crit Care 2009;24:364-370.

53 Prakash R, Mullen KD: Mechanisms, diagnosis and management of hepatic encephalopathy. Nat Rev Gastroenterol Hepatol 2010;7:515-525.

54 Bajaj JS, et al: Review article: the design of clinical trials in hepatic encephalopathy - an International Society for Hepatic Encephalopathy and Nitrogen Metabolism (ISHEN) consensus statement. Aliment Pharmacol Ther 2011;33:739-747.
Bittencourt PL, et al: Intensive care management of patients with liver disease: proceedings of a single-topic conference sponsored by the Brazilian Society of Hepatology. Arq Gastroenterol 2015;52:55-72.

56 Watanabe A, et al: Clinical efficacy of lactulose in cirrhotic patients with and without subclinical hepatic encephalopathy. Hepatology 1997;26:1410-1414.

57 Bass NM, et al: Rifaximin treatment in hepatic encephalopathy. N Engl J Med 2010;362:1071-1081.

58 Gluud LL, et al: Branched-chain amino acids for people with hepatic encephalopathy. Cochrane Database Syst Rev 2017;5:CD001939.

59 Goh ET, et al: Flumazenil versus placebo or no intervention for people with cirrhosis and hepatic encephalopathy. Cochrane Database Syst Rev 2017;7: CD002798.

60 Dalal R, et al: Probiotics for people with hepatic encephalopathy. Cochrane Database Syst Rev 2017; 2:CD008716.

61 Bajaj JS, et al: Fecal microbiota transplant from a rational stool donor improves hepatic encephalopathy: a randomized clinical trial. Hepatology 2017;66:17271738.

62 Khungar V, Poordad F: Hepatic encephalopathy. Clin Liver Dis 2012;16:301-320.

63 Katsounas A, et al: Altered expression of SHIP, a Tolllike receptor pathway inhibitor, is associated with the severity of liver fibrosis in chronic hepatitis $\mathrm{C}$ virus infection. J Infect Dis 2011;204:1181-1185.

64 Piano S, et al: Assessment of Sepsis-3 criteria and quick SOFA in patients with cirrhosis and bacterial infections. Gut 2017;DOI: 10.1136/gutjnl-2017-314324.

65 Jalan R, et al: The CLIF Consortium Acute Decompensation score (CLIF-C ADs) for prognosis of hospitalised cirrhotic patients without acute-on-chronic liver failure. J Hepatol 2015;62:831-840.

66 Lange CM, Moreau R: Immunodysfunction in acuteon-chronic liver failure. Visc Med 2018;34:DOI: 10.1159/000488690.

67 Fernández J, et al: Prevalence and risk factors of infections by multiresistant bacteria in cirrhosis: a prospective study. Hepatology 2012;55:1551-1561.

68 Fernández J, et al: Bacterial infections in cirrhosis: epidemiological changes with invasive procedures and norfloxacin prophylaxis. Hepatology 2002;35:140-148.

69 Gustot T, et al: Impact of infection on the prognosis of critically ill cirrhotic patients: results from a large worldwide study. Liver Int 2014;34:1496-1503.

70 Arabi YM, et al: Antimicrobial therapeutic determinants of outcomes from septic shock among patients with cirrhosis. Hepatology 2012;56:2305-2315.

71 Gustot $T$, et al: Invasive aspergillosis in patients with severe alcoholic hepatitis. J Hepatol 2014;60:267-274.

72 Singer M, et al: The Third International Consensus Definitions for Sepsis and Septic Shock (Sepsis-3). JAMA 2016;315:801-810.

73 Shankar-Hari M, et al: Developing a new definition and assessing new clinical criteria for septic shock: for the third international consensus definitions for sepsis and septic shock (Sepsis-3). JAMA 2016;315:775-787.

74 Seymour CW, et al: Assessment of clinical criteria for sepsis: for the third international consensus definitions for sepsis and septic shock (Sepsis-3). JAMA 2016;315: 762-774.

75 Engelmann C, Berg T: Management of infectious complications associated with acute-on-chronic liver failure. Visc Med 2018;34:DOI: 10.1159/000491107.

76 Vincent JL, Moreno R, Takala J, et al: The SOFA (Sepsis-related Organ Failure Assessment) score to describe organ dysfunction/failure. Intensive Care Med 1996;22:707-710. 